02.09.-03.09.2016

Birmingham

UK

................

08.09.-10.09.2016

Leipzig

Germany

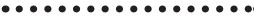

08.09.-10.09.2016

Magdeburg

Germany

•................

16.09.-18.09.2016

Erfurt

Germany

17.09.2016

Erfurt

Germany

C.....0.0....

21.09.-24.09.2016

Hamburg

Germany

23.09.-25.9.2016

Bad Wildungen

Germany

…...............

24.09.2016

Bad Wildungen

Germany

•..............

06.10.-08.10.2016

Essen

Germany

................

Innsbruck

Austria

................

07.10.-11.10.2016

Copenhagen

Denmark

08.10 .2016

Innsbruck

Austria

..................

14.10.-16.10.2016

Memmingen

Germany

15.10 .2016

Memmingen

Germany

..................

14.10.-18.10.2016

Leipzig

Germany

.................

17.10.-19.10.2016

Dubai

United Arab Emirates
Symposium 204 - Clinical Hepatology Practice in 2016:

From Science to Therapy

8. Kongress der Mitteldeutschen Chirurgenvereinigung

20. Chirurgische Forschungstage

3-tägiger Grundkurs Sedierung für Assistenzpersonal

(nach Curriculum der DEGEA/IVEPA)

1-tägiger Refreshkurs Sedierung für Assistenzpersonal

(nach Curriculum der DEGEA/IVEPA)

Viszeralmedizin 2016

3-tägiger Grundkurs Sedierung für Assistenzpersonal

(nach Curriculum der DEGEA/IVEPA)

1-tägiger Refreshkurs Sedierung für Assistenzpersonal

(nach Curriculum der DEGEA/IVEPA)

25. Jahrestagung der Deutschen Transplantationsgesellschaft

3-tägiger Grundkurs Sedierung für Assistenzpersonal

(nach Curriculum der DEGEA/IVEPA)

\section{ESMO 2016 Congress}

1-tägiger Refreshkurs Sedierung für Assistenzpersonal

(nach Curriculum der DEGEA/IVEPA)

3-tägiger Grundkurs Sedierung für Assistenzpersonal

(nach Curriculum der DEGEA/IVEPA)

1-tägiger Refreshkurs Sedierung für Assistenzpersonal

(nach Curriculum der DEGEA/IVEPA)

Jahrestagung der Deutschen Gesellschaft, Österreichischen und Schweizerischen Gesellschaften für Hämatologie und Medizinische Onkologie

3rd World Congress on Hepatitis and Liver Diseases
Information: www.falk-foundation-symposia.org

Information: www.mdcv-kongress.de

Information: www.forschungstage2016.de

Information: www.reanitrain.de

Information: www.reanitrain.de

Information: www.viszeralmedizin.com

Information: www.reanitrain.de

Information: www.reanitrain.de

Information: www.dtg2016.de

Information: www.reanitrain.de

\section{Information:}

www.esmo.org/Conferences/ESMO-2016-Congress

Information: www.reanitrain.de

Information: www.reanitrain.de

Information: www.reanitrain.de

Information: www.haematologie-onkologie-2016.com

Information: http://hepatitis.omicsgroup.com

\title{
KARGER
}


0.0 .010

Hamburg

Germany

.................

21.10.-22.10.2016

Lucerne

Switzerland

.................

22.10.2016

Munich

Germany

•...............

24.10.-27.10.2016

Augsburg

Germany

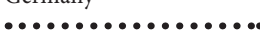

27.10.-29.10.2016

Mannheim

Germany

.................

04.11.-05.11.2016

Hamburg

Germany

05.11 .2016

Aachen

Germany

12.11 .2016

Kiel

Germany

..................

14.11.-17.11.2016

Düsseldorf

Germany

.................

17.11.-19.11.2016

Hamburg

Germany

.................

17.11.-19.11.2016

Berlin

Germany

..................

17.11.-19.11.2016

Frankfurt/M.

Germany

................

25.11.-26.11.2016

Augsburg

Germany

-...................

28.11.-02.12.2016

Hamburg

Germany

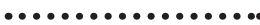

20.01.-21.01.2017

Berlin

Germany

.................

26.01.-28.01.2017

Greifswald

Germany

•..............

27.01.-30.01.2017

Amsterdam

The Netherlands

-.....................

02.02.-04.02.2017

Düsseldorf

Germany

...............

10.03.-12.03.2017

Berlin

Germany
3. DGVS-Seminar Gastroenterologische Infektionen

Symposium 205 - New Treatment Targets in Gut and Liver Diseases

1-tägiger Refreshkurs Sedierung für Assistenzpersonal

(nach Curriculum der DEGEA/IVEPA)

18. Intensivkurs Gastroenterologie

DGVS-Seminar Onkologische Gastroenterologie - Modul 2

24. Endo Club Nord

Falk Gastro-Forum - Hepato-/Gastroenterologie zwischen Wissenschaft und Praxis. Was bleibt - was kommt?

Falk Gastro-Forum - Zeitenwende in der Therapie der CED?

Medica Education Conference 2016: Heute die Medizin von morgen erfahren

3. DGVS-Seminar Neurogastroenterologie und Motilität

13. AlO-Herbstkongress - Update Medical Oncology

2016 - 32nd Annual Conference of the German Association for the Study of Obesity (DAG)

endoupdate 2016

XX. Hamburger Gastrowoche

11. Jahrestagung der Gesellschaft für Gastroenterologie und Hepatologie in Berlin und Brandenburg

37. Jahrestagung des Deutschen Pankreasclubs

ECCO 2017 - European Cancer Congress

19. Internationales Endoskopie Symposium

24. Jahrestagung der Deutschen Gesellschaft für Neurogastroenterologie und Motilität
Information:

www.dgvs.de/veranstaltungen/

dgvs-seminar-gastroenterologische-infektionen/

Information: www.falk-foundation-symposia.org

Information: www.reanitrain.de

\section{Information:}

www.dgvs.de/veranstaltungen/

intensivkurs-gastroenterologie/

Information:

www.dgvs.de/veranstaltungen/

dgvs-seminar-onkologische-gastroenterologie/

Information: www.endoclubnord.de

Information: www.falkfoundation.de

Information: www.falkfoundation.de

Information: www.medica.de/mecl

\section{Information:}

www.dgvs.de/veranstaltungen/

dgvs-seminar-neurogastroenterologie-und-motilitaet/

•..............................

Information:

www.aio-portal.de/index.php/

termineveranstaltungen.html

Information: www.frankfurter-meeting.de

Information: www.endoupdate.de

Information: www.gastroclub.org/index.php?id=10

Information: www.gghbb.de

Information: www.dpc-kongress.de

Information: www.ecco-org.eu/Events/ECCO2017

\section{Information:}

www.endo-duesseldorf.com/german/registration.php

Information: www.dgvs.termin-kalender.org/ detail/1431/24-jahrestagung-der-deutschen-gesellschaft-

fuer-neurogastroenterologie-und-motilitaet 
10.03.-12.03.2017

Nuremberg

Germany

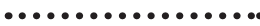

21.03.-24.03.2017

Munich

Germany

.................

24.03.-25.03.2017

Berlin

Germany

...............

30.03.-01.04.2017

Munich

Germany

.................

06.04.-08.04.2017

Berlin

Germany

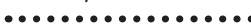

06.04.-08.04.2017

Berlin

Germany

1.................

Frankfurt/M.

Germany

.................

23.06.-24.06.2017

Hanover

Germany

24.06.2017

Wiesbaden

Germany

•.....................

28.06.-30.06.2017

Vienna

Austria

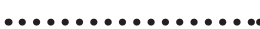

19.07.-21.07.2017

Würzburg

Germany

.................

13.09.-16.09.2017

Dresden

Germany

.................

13.10.-18.10.2017

Orlando, FL

USA

.................

03.11.-04.11.2017

Hamburg

Germany

•..............

16.11.-18.11.2017

Berlin

Germany

..................

23.11.-25.11.2017

Augsburg

Germany

................

15.03.-17.03.2018

St. Gallen

Switzerland
Bundeskongress Chirurgie - BNC, BDC, BAO gemeinsam stark

134. Kongress der Deutschen Gesellschaft für Chirurgie

11. AlO-GI-Tag - Treffen der Arbeitsgruppen des Bereichs

Gastrointestinaler Tumoren

43. Koloproktologen-Kongress (DGK)

DGE-BV meets Endoskopie Live - 47. Kongress der Deutschen

Gesellschaft für Endoskopie und Bildgebende Verfahren

Endoskopie Live meets DGE-BV

26. Kongress der Mitteldeutschen Gesellschaft für Gastroenterologie

14. HepNet Symposium

GI-Oncology 2017 - 13. Interdisziplinäres Update

58. Österreichischer Chirurgenkongress

94. Jahrestagung der Vereinigung der Bayerischen Chirurgen e.V. (VBC)

Viszeralmedizin 2017

Gastro 2017 - ACG-WGO World Congress of Gastroenterology

25. Endo Club Nord

14. AlO-Herbstkongress - Update Medical Oncology

endoupdate 2017

4th St. Gallen International Gastrointestinal Cancer Conference
Information: www.bundeskongress-chirurgie.de

Information: www.chirurgie2017.de

Information: www.aio-portal.de

Information:

www.mcn-nuernberg.de/kalender-2017.php

Information: www.dge-bv.de

Information: www.endoskopie-live-berlin.de

Information: www.mgfg.de/kongress-2017/

\section{Information:}

www.deutsche-leberstiftung.de/symposium/

14.-hepnet-symposium

\section{Information:}

www.gi-oncology.de/html/weitere_termine.html

Information: www.chirurgenkongress.at

Information: www.mcn-nuernberg.de

Information: www.dgvs.de

Information: info@worldgastroenterology.org

Information: www.endoclubnord.de/de/

Information: www.aio-portal.de

Information: www.endoupdate.de

Information: www.oncoconferences.ch 